ŹróDŁA, MATERIAŁY, MisCELLANEA

http://dx.doi.org/10.15762/ZH.2018.45

RAFAE KUBICKI

(Uniwersytet Gdański)

\title{
WYKAZ CZYNSZÓW I SŁUŻB OKRĘGU BARCIANY Z OKOŁO POŁOWY XV WIEKU
}

Słowa kluczowe: zakon krzyżacki w Prusach, gospodarka, osadnictwo, źródła gospodarcze, spisy czynszowe

Badania nad obrazem sieci osadniczej państwa zakonu krzyżackiego w Prusach wymagają uwzględnienia możliwie pełnego zestawu zachowanych przekazów źródłowych. Wśród nich ważne miejsce, obok przywilejów fundacyjnych, zajmują wykazy czynszowe, obejmujące spisy wsi płacących czynsz na rzecz zakonu, i zestawienia służb wojskowych, do których zobowiązani byli m.in. właściciele dóbr na prawie chełmińskim, magdeburskim i pruskim.

Przykładem tego rodzaju źródel jest prezentowany tu wykaz czynszów i służb z okręgu Barciany, powstały ok. połowy XV w. Przekaz jest niedatowany, jednak analiza zawartych w nim danych szczegółowych pozwala umiejscowić go po barciańskim wykazie czynszowym z 1437 r. Prawdopodobnie powstał pod koniec lat czterdziestych XV w. (po 1447 r.). Mogą wskazywać na to następujące dane: w $1437 \mathrm{r}$. wieś Siniec płaciła czynsz z całej powierzchni 50 łanów, względnie z 44 łanów $^{1}$, a w publikowanym tu wykazie odnotowano, że nie płaciła czynszu ani w pieniądzu, ani w zbożu od trzech lat (k. 3v). Z kolei w Garbnie w 1437 r. płacono czynsz z wszystkich 23 radeł ziemi ${ }^{2}$, natomiast w prezentowanym tu wykazie zaznaczono, że poza 20 radłami, z których płacono czynsz, były jeszcze dwa puste od 10 lat (k. 1r). Z kolei wieś Radzieje w 1437 r. miała płacić czynsz z 50 łanów ${ }^{3}$ lub nie płacić go w ogóle. ${ }^{4}$. W publikowanym tu spisie wskazano, że wieś od trzech lat nie płaciła czynszu ani w pieniądzu, ani w zbożu (k. 3v).

${ }^{1}$ Das Grosse Zinsbuch des Deutschen Ritterordens (1414-1438) (dalej cyt. GZB), hrsg. v. Peter G. Thielen, Marburg 1958, s. 53, 68.

${ }^{2}$ GZB, s. 67.

${ }^{3}$ GZB, s. 53.

${ }^{4}$ GZB, s. 68 . 
Omawiany przekaz stanowi jednocześnie dopełnienie wcześniejszych wykazów czynszowych z lat 1422 i 1437, które wydane zostały w ramach tzw. wielkiej księgi czynszowej. Zawarte $w$ nim informacje na temat stanu osadnictwa (wsie czynszowe) i służb wojskowych obejmują obok prokuratorstwa w Barcianach również dobra służebne w ówczesnej zachodniej części prokuratorstwa leckiego (giżyckiego) ${ }^{6}$. Źródło to może być przydatne w dalszych pracach nad rekonstrukcją osadnictwa okolic Barcian i Kętrzyna ok. połowy XV w. ${ }^{7}$ Uwzględniając wcześniejsze przekazy, można dzięki niemu prześledzić zmiany w stanie zagospodarowania wsi oraz rodzaju, liczbie i rozmieszczeniu dóbr służebnych na terenie poszczególnych osad.

Omawiany wykaz przechowywany jest obecnie w Geheimes Staatsarchiv Preußischer Kulturbesitz (Tajne Archiwum Pruskich Dóbr Kultury) w Berlinie Dahlem (GStA PK), w obrębie zbiorów dawnego archiwum zakonu krzyżackiego. Umieszczono go wśród listów (Ordensbriefarchiv) pod sygnaturą 28754. W drukowanym regestariuszu korespondencji zakonu opisano go jako: "Rejestr czynszu i służb okręgu barciańskiego" (Register des Zinses und Dienste des Gebietes Barthen).

Źródło to spisano na kartach papierowych złożonych w ten sposób, że tworzą format dudki o wymiarach $9,5 \times 25,5 \mathrm{~cm}$. Tekst spisano w języku niemieckim, minuskułą gotycką, pismem czytelnym z użyciem nielicznych abrewiacji. W tekście znajduje się tylko kilka skreśleń. Kolejne karty mają foliację od 1 do $6 r$. W przygotowaniu tekstu do druku posłużono się częściowo zasadami

${ }^{5}$ GZB, s. 27-29, 66-74.

${ }^{6}$ Wcześniejsze z 1417 i 1437 r. zawarte są w GZB, s. 4, 68. Na temat osadnictwa na tym terenie do 1454 r. zob. Lothar WEBER, Preussen vor 500 Jahren in culturhistorischer, statistischer und militarischer Beziehung nebst Special-Geographie, Danzig 1878, s. 501-502, 510-511; Grzegorz BIAŁUŃsKI, Osadnictwo regionu Wielkich Jezior Mazurskich od XIV do początku XVIII wieku - starostwo leckie (giżyckie) i ryńskie, Olsztyn 1996, s. 28-30.

${ }^{7}$ Temat ten podejmowany był już wielokrotnie w literaturze przedmiotu, jednak bez uwzględnienia prezentowanego tu przekazu, przykładowo zob. Carl BECKHERRN, Beiträge zur Topographie und Statistik des ehemaligen Amtes Rastenburg, Altpreußische Monatsschrift, Jg. 18: 1881, s. 398-437, 513-537; Wojciech KęTRZYŃski, O ludności polskiej w Prusiech niegdyś krzyżackich, Lwów 1882; Martin Rousselle, Das Siedlungswerk des Deutschen Ordens im Lande Gerdauen, Altpreußische Forschungen, Jg. 6: 1929, s. 220-255; Hans i Gertrud Mortensen, Die Besiedlung des nordöstlichen Ostpreußens bis zum Beginn des 17. Jahrhunderts, Teil 1: Die preußisch-deutsche Siedlung am Westrand der Großen Wildnis um 1400, Leipzig 1937; Karol GóRsKI, Marian Arszý́ski, Barciany. Dzieje zamku i ziemi do połowy XV wieku, Olsztyn 1967, s. 24-31; Lidia WAKUluK, Osadnictwo i sieć parafialna kętrzyńskiego okręu prokuratorskiego w XIV-XV wieku, Komunikaty Mazursko-Warmińskie, 1976, nr 3 (133), s. 375-395; Tadeusz Swat, Dzieje wsi, [in:] Kętrzyn. Z dziejów miasta i okolic, red. Andrzej WaKar, Olsztyn 1978, s. 161-242.

${ }^{8}$ Regesta historico-diplomatica Ordinis S. Mariae Theutonicorum 1198-1525 (dalej cyt. RHDO), Pars I, vol. 3: (1511-1525), bearb. v. Erich JoAchim, hrsg. v. Walther HubATsch, Göttingen 1973, nr 28754, s. 525. 
zawartymi w instrukcji wydawniczej Adama Wolffa ${ }^{9}$. W celu ukazania podstawy źródłowej użyto z instrukcji symbolu: / - -/ skreślenie, $<>$ dopisek. Abrewiacje rozwiązano bez zaznaczania tego w tekście. W edycji zastosowano następujące skróty: d. - denarius (fenig, denar), f. - ferto/firdung (wiardunek), mr. - mark (grzywna), sc. - scot (skojec), sol. - solidus (szeląg), sch. - scheffel (korzec), lb. - libra (funt). Obowiązujące ówcześnie w państwie krzyżackim miary zbożowe to: 1 korzec $(55,0$ litrów $)=4$ wiertle $=16$ miarek, a system rachunkowo-pieniężny: 1 grzywna $=4$ wiardunki $=24$ skojce $=60$ szelągów (solidów) $=720$ fenigów (denarów). Oszczędność miejsca w druku zadecydowała o nieoddawaniu oryginalnego układu tekstu, lecz zastąpieniu go wprowadzonym przez wydawcę uporządkowaniem graficznym.

\section{REJESTR CZYNSZÓW I SŁUŻB OKRĘGU BARCIANY \\ $Z$ OKOŁO POŁOWY XV WIEKU}

Oryginat: GStA PK, XX. HA, Ordensbriefarchiv nr 28754.

Regest: RHDO, Pars 1, vol. 3 (1511-1525), s. 525.

[k. 1r]

Barthen

Dysse nochgeschreben prewschen hoken zeyn im gebitte Barthen ${ }^{10}$ unde geben czehenden, weysen, rocken, gerste, haber.

Item welche gebower haben 2 hoken, dy yo vom hoken $3 \mathrm{sch}$. weysen, $3 \mathrm{sch}$. rocken, $3 \mathrm{sch}$. gerste, $11 \mathrm{sch}$. habir.

Item dy zelbigen prewschen gebawer geben adder dinstgelt yo von czwehn hoken 1 gutten $\mathrm{f}$. unde dis nochgeschreben gelt ist alz noch guttim gelde geschreben.

Item dy zelbigen gebawer geben sorgalia ${ }^{11}$ adder wartgelt yo von czwehen hoken 4 sol.

Item welche prewsche gebawer haben 3 hoken, dy geben $41 / 2$ sch. weysen, $41 / 2$ sch. rocken, $41 / 2$ sch. gerste, $161 / 2$ sch. haber.

Item welche prewsche gebawer haben $11 / 2$ hoken, dy geben 2 sch. weysen 1 firtil, 2 sch. rocken 1 firtil, 2 sch. gerste 1 firtil, 8 sch. haber 1 firtil.

${ }^{9}$ Adam WolfF, Projekt instrukcji wydawniczej dla pisanych źródeł historycznych do połowy XVI wieku, Studia Źródłoznawcze, t. 1: 1957, s. 155-184. Nowe wydanie instrukcji zob. Janusz TANDECKI, Krzysztof KopIŃsKi, Edytorstwo źródet historycznych, Warszawa 2014, s. 328-354.

${ }^{10}$ Barciany.

${ }^{11}$ Sorgalia (lit. sargs- strażnik i alga opłata) wartowe, opłata wnoszona przez chłopów pruskich zob. Wiesław DŁUgokęCKI, Uwagi o genezie i rozwoju wczesnośredniowiecznych Prus do początków XIII w., Pruthenia, t. 2: 2006, s. 39. 
Meladen ${ }^{12}$ Item in deme dorffe zeyn 12 hoken do von zeyn 4 hoken dis joer vorbrant unde 2 hoken wuste.

Smedeyn ${ }^{13}$ Item in dem dorffe zeyn 14 hoken do von zeyn czwene hoken vorkowfft.

Rastekaym ${ }^{14}$ Item in dem dorffe zeyn 6 hoken do von zeyn 3 hoken vorgeben. Losekaym ${ }^{15}$ Item do zeyn 6 hoken do von zeyn 4 vorkowfft.

Laggarben ${ }^{16}$ Item do zeyn 20 hoken besatczt unde do geben 2 hoken dis joer nicht zehenden wen zy wol 10 yoer han wuste gelegen.

[k. 1v]

Swilgynnen ${ }^{17}$ Item do zeyn 13 hoken besatczt.

Moddegerben ${ }^{18}$ Item do zeyn 15 hoken do von zeyn 7 hoken vorgeben unde geben nicht slowszim unde mussen gras sloen iclicher 4 1/2 morgen unde geben sorgalia.

Zaynsegreben ${ }^{19}$ Item do zeyn 19 hoken besatczt unde geben nicht slowszim unde sloen gras iclicher 4 1/2 morgen unde geben sorgalia.

Kollekaym ${ }^{20}$ Item do zeyn 8 hoken besatczt.

Marclawcken ${ }^{21}$ Item do zeyn 4 hoken besatczt.

Boggelawcken ${ }^{22}$ Item do zeyn 12 hoken besatczt.

Cleyne Kemlawcken ${ }^{23}$ Item do zeyn 12 hoken besatczt.

Plunekaym ${ }^{24}$ Item do zeyn 8 hoken besatczt.

Summa der hoken $47^{\text {a }}$ und $100^{\text {a }}$ hoken obiral do von zeyn 16 hoken vorgeben unde vorkowfft unde 2 hoken wuste, alz hir voer berurt ist, unde zeyn $31^{\text {b-und }}$ $100^{-b}$ czinshafftik.

a- -a Tekst nadpisany.

b- -b Tekst nadpisany.

${ }^{12}$ Melejdy (niem. Mehleden), pow. bartoszycki, gm. Sępopol.

${ }^{13}$ Smodajny (niem. Schmodehnen), pow. bartoszycki, gm. Sępopol.

${ }^{14}$ Rastekaym, osada zaginiona, część wsi Rädtkeim w okręgu gierdawskim zob. H. i. G. MoRTENSEN, op.cit., s. 192. Wskazywałby na to geograficzny układ spisu. Idąc od północy, mamy Melejdy, dalej na południowy wschód Smodajny i Łoskajmy, a na północ od nich Rädtkeim.

${ }^{15}$ Łoskajmy (niem. Losskeim), pow. bartoszycki, gm. Sępopol.

${ }^{16}$ Garbno (niem. Lamgarben), pow. kętrzyński, gm. Korsze.

${ }^{17}$ Silginy (niem. Silliginnen), pow. kętrzyński, gm. Barciany.

${ }^{18}$ Modgarby (niem. Modgarben), pow. kętrzyński, gm. Barciany.

${ }^{19}$ Gęsie Góry (niem. Sansgarben), pow. kętrzyński, gm. Barciany.

${ }^{20}$ Kolkiejmy (niem. Kollkeim), pow. kętrzyński, gm. Srokowo.

${ }^{21}$ Markławka (niem. Marklack), pow. kętrzyński, gm. Barciany.

${ }^{22}$ Podławki (niem. Podlacken), pow. kętrzyński, gm. Barciany.

${ }^{23}$ Kiemławki Małe (niem. Klein Kämlack), pow. kętrzyński, gm. Barciany.

${ }^{24}$ Plinkajmy Małe (niem. Plienkeim), pow. kętrzyński, gm. Barciany, osada opuszczona. 
Item summa des slowszims adder dinst geldis $12 \mathrm{mr}$. unde $1 \mathrm{f}$. Item summa der sorgalia adder wartgeldis $4 \mathrm{mr}$. unde $4 \mathrm{sc}$. Item summa des czehenden primo etc. Item 3 leste rocken $3 \mathrm{sch}$. Item 3 leste weysen $3 \mathrm{sch}$. Item 3 leste gerste 3 sch. Item 11 leste habir 6 sch.

[k. 2r]

Dysse nochgeschreben daz zeyn dy prewschen freien

Primo zcu Meladen zeyn 2 prewsche dinste. Item zcu Smedeyn zeyn 3 prewsche dinste. Item zcu Rastekaym zeyn 2 prewsche dinste. Item zcu Losekaym zeyn 3 prewsche dinste. Item zcu Llaggarben zeyn 5 prewsche dinste. Item zcu Swilgynnen zeyn 2 prewsche dinste. Item zcu Solkeniken ${ }^{25}$ zeyn 8 prewsche dinste. Item zcu Kollekaym zeyn 5 prewsche dinste. Item zcu Jegelawcken ${ }^{26}$ zeyn 11 prewsche dinste. Item zcu Rodelekaym ${ }^{27}$ zeyn 7 prewsche dinste. Item zcu Merclawcken zeyn 2 prewsche dinste. Item zcu Bogglawcken zeyn 5 prewsche dinste. Item zcu Grose Kelmelawcken ${ }^{28}$ zeyn 3 prewsche dinste. Item zcu Cleyne Kelmlawcken zeyn 5 prewsche dinste. Item zcu Plunekaym zeyn 6 prewsche dinste. Item zcu Crymlawcken ${ }^{29}$ zeyn 5 prewsche dinste. Item zcu Kudewin $^{30}$ zeyn 2 prewsche dinste.

Summa der dinste 76 prewsche dinste.

Item dy zelbigen prewschen freien geben sorgalia adder wartgelt jo vom /-hoken-/ dinste 1 loet adder $1 / 2$ geringen $\mathrm{f}$.

Summa $41 / 2 \mathrm{mr}$. unde 1 loet gut gelt.

Dysse nochgeschreben zeyn magdenburgsche dinste.

Primo zcu Wayninikaym ${ }^{31}$ ist 1 magdenburgsche dinste. Item Schordennen ${ }^{32}$ hoff do zeyn 2 magdenburgsche dinste. Item zcu Rodelen ${ }^{33}$ do zeyn 2 magdenburgsche dinste. Item zcu Plactlawcken ${ }^{34}$ ist 1 magdenburgsche dinste.

[k. 2v]

Dysse nochgeschreben zeyn kolmische dinste unde geben jo vom dinste $1 \mathrm{sch}$. weysen, $1 \mathrm{sch}$. rocken unde $1 \mathrm{lb}$. wachß unde $5 \mathrm{~d}$. zcur oerkunde.

${ }^{25}$ Solkieniki (niem. Solknick), pow. kętrzyński, gm. Barciany.

${ }^{26}$ Jegławki (niem. Jäglack), pow. kętrzyński, gm. Srokowo.

${ }^{27}$ Rodelekaym, osada zaginiona.

${ }^{28}$ Kiemławki Wielkie (niem. Grosse Kämlack), pow. kętrzyński, gm. Barciany.

${ }^{29}$ Krymławki (niem. Krimlack), pow. kętrzyński, gm. Barciany.

${ }^{30}$ Kudwiny (niem. Kudewinnen), pow. kętrzyński, gm. Barciany.

${ }^{31}$ Wanikajmy (niem. Woninkeim), pow. bartoszycki, gm. Sępopol.

${ }^{32}$ Schordennen Hoff (Schordeyn), dobra zaginione.

${ }^{33}$ Rodele (niem. Rodehlen), pow. kętrzyński, gm. Barciany.

${ }^{34}$ Płatławki (niem. Platlack), pow. kętrzyński, gm. Barciany. 
c-Primo zcu Moddegarben zeyn 3 kolmische dinste. Item eyn magdenburgsch dinste. Item 1 prewsche dinst. ${ }^{-c \text { d }}$ Item zcu Schandaw ${ }^{35}$ zeyn 3 kolmische dinste. Item 1 magdenburgsche dinst ${ }^{-\mathrm{d}}$. Item zcu Schandlawcken ${ }^{36}$ ist eyn platen dinst. Item zcu Samsegerben ${ }^{37}$ ist 1 colmisch dinst. Item zcu Jegelawcken ist eyn colmisch dinst. Item zcur Wickraw ${ }^{38} 2$ colmische dinste. Item zcum Bomgarthen ${ }^{39}$ zeyn 4 colmische dinste. Item dozelbist 1 platen dinst. Item zcu Peetczschen ${ }^{40}$ ist 1 colmisch dinst. Item Thomas Salmon 1 colmischen dinst. Item zcu Grose Kelmlawcken 1 colmisch. Item Joest Camplawcken 1 colmischen dinst. Item Hans Camplawcken 1 colmischen dinst. Item Petermansdorff ${ }^{41} 1$ kolmischen dinst. Item Dombeyen ${ }^{42} 1$ colmischen dinst.

\section{Leetczen ${ }^{43}$}

Item Alde Sterlawcken ${ }^{44} 14$ colmische dinste. Item Nowe Sterlawcke ${ }^{45}$ dinet noch nicht. Item Mertenhayn ${ }^{46} 1$ platen dinst. Item Partczschin ${ }^{47} 3$ colmische dinst. Item Jomkendorff ${ }^{48} 1$ platen dinst. Item Cronaw ${ }^{49} 1$ colmischen dinst. Item Ansehen ${ }^{50} 2$ colmische dinste. Item Pogriselawcken ${ }^{51} 1$ colmischen dinst. Item Dringenfort ${ }^{52} \mathrm{dy}$ stad hat 1 platen dinst unde gibbet $2 \mathrm{sch}$. weysen, $2 \mathrm{sch}$. rocke, $2 \mathrm{lb}$. wachs unde $10 \mathrm{~d}$. [k. 3r] Item Gorlitcz ${ }^{53}$ daz dy burger zcu Rastenburg $^{54}$ haben 1 platen dinst.

Summa der colmischen unde platen dinste 48 dinste unde geben obiral 1 steyn unde $8 \mathrm{lb}$. kolmisch wachß.

c--c, d- -d Dopisek $z$ prawej strony: eyne alleyne.

${ }^{35}$ Skandawa (niem. Skandau), pow. kętrzyński, gm. Barciany.

${ }^{36}$ Skandławki (niem. Skandlack), pow. kętrzyński, gm. Srokowo.

${ }^{37}$ Gęsie Góry zob. przypis 19.

${ }^{38}$ Wikrowo (niem. Wickerau), pow. kętrzyński, gm. Srokowo.

${ }^{39}$ Ogródki (niem. Baumgarten), pow. kętrzyński, gm. Barciany.

${ }^{40}$ Piecki (niem. Peitschendorf), powiat Mrągowo.

${ }^{41}$ Pieszewo (niem. Petermanss), pow. kętrzyński, gm. Barciany.

${ }^{42}$ Dębiany (niem. Dombehnen), pow. kętrzyński, gm. Barciany.

${ }^{43}$ Okręg lecki (giżycki).

${ }^{44}$ Sterławki Wielkie (niem. Gross Stürlack), pow. giżycki, gm. Ryn.

${ }^{45}$ Sterławki Małe (niem. Klein Stürlack), pow. giżycki, gm. Ryn.

${ }^{46}$ Martiany (niem. Mertenheim), pow. kętrzyński, gm. Kętrzyn.

${ }^{47}$ Parcz (niem. Partsch), pow. kętrzyński, gm. Kętrzyn.

${ }^{48}$ Jankowo (niem. Jankendorf), pow. kętrzyński, gm. Kętrzyn.

${ }^{49}$ Kronowo (niem. Kronau), pow. giżycki, gm. Ryn.

${ }^{50}$ Mażany (niem. Masehnen), pow. kętrzyński, gm. Kętrzyn.

${ }^{51}$ Gryzławki (niem. Grieslack), pow. kętrzyński, gm. Kętrzyn.

${ }^{52}$ Dryfort (niem. Drengfurth), obecnie Srokowo.

${ }^{53}$ Gierłoż (niem. Görlitz), pow. kętrzyński, gm. Kętrzyn.

${ }^{54}$ Miasto Kętrzyn. 
Item dyzelbigen kolmner geben $8 \mathrm{sc}$. colmische pfennige zcur oerkunde. Item geben dyzelbigen colmner kolmisch korn. Item 1 last weysen minus $12 \mathrm{sch}$. Item 1 last rocken minus $12 \mathrm{sch}$.

Dyssen nochgeschreben czins hebet der komptur von Brandenburg ${ }^{55}$ awß dem ampte zcu Barthenburgk primo etc.

Wenden $^{56}$

Item das dorff hot 53 czinszhuben yo dy hube czinset 1/2 mr. Summa 26 1/2 mr. Item czinsen zy von iclich huben 1 firtil weisen, 1 firtil rocken. Summa 13 sch. 1 firtil weysen und rocken owch zo veel. Item czinsen zy von iclich huben 3 huner. Summa 150 unde 9 huner. Item der schultcze dozelbist czinset 1 sch. weysen, $1 \mathrm{sch}$. rocken. Item der kretczschmer dozelbist czinset $3 \mathrm{mr}$. unde $3 \mathrm{lb}$. pffeffer unde der ist wuste.

Rosentael $^{57}$

Item daz dorff hot 50 huben yo dy hube czinset $1 / 2 \mathrm{mr}$. Summa $25 \mathrm{mr}$. Item geben zy von iclicher huben 1 firtil weysen, 1 firtil rocken. Summa 12 1/2 sch. weysen unde $121 / 2 \mathrm{sch}$. rocken. Item czinsen zy von iclicher huben 2 huner. Summa 100 huner. Item der schultcze czinset $1 \mathrm{sch}$. weysen unde $1 \mathrm{sch}$. rocken. Item der kretczschmer dozelbest czinset $21 / 2 \mathrm{mr}$.

\section{[k. 3v]}

Heynrichsdorff ${ }^{58}$

Item daz dorff hot 54 czinsshuben yo dy hube czinset $1 / 2 \mathrm{mr}$. Summa $27 \mathrm{mr}$. Item czinßen zy von iclicher huben 1 firtil weysen, 1 firtil rocken. Summa 13 1/2 sch. weysen unde $131 / 2$ sch. rocken. Item geben zy von iclicher huben 2 huner. Summa 100 unde 8 huner. Item der schultcze dozelbist czinset 1 sch. weysen, 1 sch. rocken.

Plawensteyn ${ }^{59}$

Item daz dorff hot 50 czinshuben unde hot innen dreien joren keynen czinß gegeben noch pffennigen czins noch getreide.

Rosengarthen ${ }^{60}$

${ }^{55}$ Komtur w Brandenburgu (Pokarminie).

${ }^{56}$ Winda (niem. Wenden), pow. kętrzyński, gm. Barciany.

${ }^{57}$ Stara Różanka (niem. Alt Rosenthal), pow. kętrzyński, gm. Kętrzyn.

${ }^{58}$ Heynrichsdorff, najprawdopodobniej tożsama z wsią Silec (niem. Schülzen), pow. kętrzyński, gm. Srokowo, na co wskazuje taka sama powierzchnia obu osad (54 łany). Jej pierwotna nazwa związana była prawdopodobnie z działalnością lokacyjną krzyżackiego prokuratora w Barcianach Henryka von Plauena (1402-1406), zob. K. GóRSKI, M. ARszyŃski, op.cit., s. 25.

${ }^{59}$ Siniec (niem. Gross Blaustein), pow. kętrzyński, gm. Srokowo.

${ }^{60}$ Radzieje (niem. Rosengarten), pow. węgorzewski, gm. Węgorzewo. 
Item daz dorff hot 50 huben unde hot in dreien joren keynen czinß gegeben noch pfenige czins noch getreide.

Wopelawcken ${ }^{61}$

Item daz dorff hot 51 huben yo dy hube czinset 13 sc. Summa 27 1/2 mr. 1/2 f. Item geben zy von der huben 1 sch. weysen, 1 sch. rocken. Summa $13 \mathrm{sch}$. weysen minus 1 firtil $13 \mathrm{sch}$. rocken minus 1 firtil. Item geben zy von der huben 2 huner. $<$ Summa $>100$ unde 2 huner. Item der schultcze dozelbist czinset $1 \mathrm{sch}$. weysen, $1 \mathrm{sch}$. rocken.

Swartczensteyn ${ }^{62}$

Item daz dorff hat 41 huben icliche hube czinset $14 \mathrm{sc}$. Summa $24 \mathrm{mr}$. minus $2 \mathrm{sc}$. Item von iclicher huben czinsen zy 1 firtil weysen, 1 firtil rocken. Summa 10 sch. 1 firtil weysen, 10 sch. 1 firtil rocken.

[k. 4r]

Item Swartczensteyn /-zeyne-/ czinßen zy von iclicher huben 2 huner. Summa 1 schok 22 huner. Item der schultcze dozelbist czinset $1 \mathrm{sch}$. weysen, $1 \mathrm{sch}$. rocken. Item der molner czinset $4 \mathrm{mr}$. unde 4 vette genße. Item der kretczschmer dozelbist czinset $3 \mathrm{mr}$.

Dowben $^{63}$

Item do zeyn czwene kretczschmer. Item der eyne czinset $4 \mathrm{mr}$. Item der ander czinsset $4 \mathrm{mr}$.

Swilgynnen

Item der kretczmer czinset 3 1/2 mr.unde 1/2 schok huner. Item Kirstan Dawgil czinset $1 \mathrm{mr}$. vor 1 hube.

Laggarben

Item do zeyn czwene kretczschmer. Item der obirste kretschmer czinset $4 \mathrm{mr}$. minus 1f. unde 1/2 schok huner. Item der niddirste kretczschmer czinset $4 \mathrm{mr}$. unde $1 \mathrm{f}$. unde 12 vette genße.

Smedeyen

Item der molner czinßet $5 \mathrm{mr}$. unde 1/2 schok huner. Item der kretczschmer czinset $21 / 2 \mathrm{mr}$.

Jegelawcken

Item der molner czinset $6 \mathrm{mr}$. 1/2 schok huner.

${ }^{61}$ Wopławki (niem. Woplaucken), pow. kętrzyński, gm. Kętrzyn.

${ }^{62}$ Czerniki (niem. Schwarzstein), pow. kętrzyński, gm. Kętrzyn.

${ }^{63}$ Doba (niem. Doben), pow. giżycki, gm. Giżycko. 
Mergentael $^{64}$

Item das dorff hot 50 huben yo dy hube czinset $1 / 2 \mathrm{mr}$. Summa $25 \mathrm{mr}$. Item geben zy yo von der huben $5 \mathrm{sch}$. pffluc habir. Summa 2 leste $5 \mathrm{sch}$. haber. Item geben zy yo von der huben 2 huner. Summa 100 huner. Item der schultcze gibbet $1 \mathrm{sch}$. weysen, $1 \mathrm{sch}$. rocken. Item der kretczschmer czinßet $3 \mathrm{mr}$. unde $3 \mathrm{lb}$. pfeffer.

[k. 4v]

Dringenfort daz dorff ${ }^{65}$

Item daz dorff hat 55 huben yo dy hube czinset $1 / 2 \mathrm{mr}$. Summa $271 / 2 \mathrm{mr}$. Item geben zy von 2 huben 5 sch. pffluc habir. Summa 2 leste, 17 1/2 sch. Item geben zy von iclicher huben 3 huner. Summa 165 huner. Item der schultcze gibbet $1 \mathrm{sch}$. weysen, $1 \mathrm{sch}$. rocken. Molner. Item der molner czinßet $24 \mathrm{mr}$. alle quatertemper $6 \mathrm{mr}$.

Dringenfort dy stad

Item dy stad czinsset $12 \mathrm{mr}$.unde $1 \mathrm{f}$. Item czinset dy stad $1 \mathrm{mr}$. unde $2 \mathrm{sc}$. vom roethawße.

Furstenaw $^{66}$

Item daz dorff hot 52 hube yo dy hube czinßet $1 / 2 \mathrm{mr}$. Summa $26 \mathrm{mr}$. Item geben zy yo von 2 huben $5 \mathrm{sch}$. pfluchabir. Summa 2 leste unde $10 \mathrm{sch}$. Item czinßen zy von der huben 2 huner. Summa 100 unde 4 huner. Iten der schultcze gibbet $1 \mathrm{sch}$. weysen unde $1 \mathrm{sch}$. rocken.

[k. 5r]

Des pflegers czins zcu Barthen

Lyßke $^{67}$

Item in der lißken zeyn 9 kretczschmer iclicher czinset $3 \mathrm{mr}$. Summa $27 \mathrm{mr}$. Item dy gemeyne czinset $9 \mathrm{mr}$. vor 12 huben waldis. Item dy gemeyne czinset $5 \mathrm{mr}$. unde $1 \mathrm{f}$. vor 7 huben ackir. Item dy gemeyne czinset $3 \mathrm{mr}$. minus $1 \mathrm{f}$. vor 2 huben unde 40 morgen. Item dy gertener czinsen 11 mr. minus $1 \mathrm{f}$.

Frowdenburg ${ }^{68}$

Item daz dorff hat 50 czinßhuben yo dy hube czinßet $1 / 2 \mathrm{mr}$. summa $25 \mathrm{mr}$. Item geben zy von iclicher huben 1 firtil weysen, 1 firtil rocken. Summa 11 1/2 sch. weysen, 11 1/2 sch. rocken. Item von iclicher huben 3 huner. Sum-

${ }^{64}$ Kosakowo (niem. Marienthal), pow. kętrzyński, gm. Srokowo.

${ }^{65}$ Dryfort (obecnie Srokowo), wieś miejska należąca do miasta Dryfort (obecnie Srokowo).

${ }^{66}$ Leśniewo (nie. Fürstenau), pow. kętrzyński, gm. Srokowo.

${ }^{67}$ „Liszka” (osada handlowa) przed zamkiem w Barcianach.

${ }^{68}$ Radosze (niem. Freudenberg), pow. kętrzyński, gm. Barciany. 
ma 150 huner. Item der schultcze dozelbist czinset $1 \mathrm{sch}$. weysen, $1 \mathrm{sch}$. rocken. Item der kretczschmer dozelbist czinßet $3 \mathrm{mr}$. unde $3 \mathrm{lb}$. pffefer.

/-Marclawcken Item daz dorff hot 28 czinsehuben yo dy hube czinßet 1/2 mr-/ Marclawcken

Item der kretczschmer czinßet $2 \mathrm{mr}$. Item der molner dozelbist czinße $7 \mathrm{mr}$. unde $1 / 2$ schok huner.

Meisterffelt ${ }^{69}$

Item daz dorff hot 28 czinßhuben yo dy hube czinßet $1 / 2 \mathrm{mr}$. Item czinßen zy vor 4 huben waltczins $1 \mathrm{mr}$. unde $81 / 2 \mathrm{sc}$. Summa $15 \mathrm{mr} .81 / 2 \mathrm{sc}$.

[k. 5v]

Item Meisterfelt geben zy von iclicher huben 1 firtil weysen, 1 firtil rocken. Summa 6 sch. weysen, 6 sch. rocken. Item geben zy von iclicher huben 3 huner. Summa 1 1/2 schock minus 6 huner. Item der schultcze gibbet 1 sch. weysen, 1 sch. rocken.

Wulffishayn $^{70}$

Item der molner czinßet 4 mr. 3 vette genße.

Pariss $^{71}$

Item dy czinßen 2 1/2 mr.waltczinß.

Pleyn $^{72}$

Item czinßen zy $11 / 2$ mr. waltczinß von den Wayputten ${ }^{73}$.

[k. 6r]

Summa summarum der prewschen hoken

Item $47^{\mathrm{e}-}$ und $100^{\text {ee }}$ hoken obiral do von zeyn 16 hoken vorgeben unde vorkawfft. Item 2 wuste hoken. Item $31^{\mathrm{f}-}$ und $100^{-\mathrm{f}}$ czinßhafftige hoken.

Summa summarum der kolmischen, magdeburgsche, platen, prewschen dinste 124 dinste.

Summa summarum des slawsims adder dinstgeldis $12 \mathrm{mr}$. guttis geldis und $1 \mathrm{f}$.

Summa summarum der sorgalia adder wartgelt $4 \mathrm{mr}$. unde $4 \mathrm{sc}$. guttis geldis.

e--e Tekst nadpisany.

f-f Tekst nadpisany.

${ }^{69}$ Gęsiki (niem. Meisterfelde), pow. kętrzyński, gm. Barciany.

${ }^{70}$ Wilczyny (niem. Wolfshagen), pow. kętrzyński, gm. Srokowo.

${ }^{71}$ Parys (niem. Paaris), pow. kętrzyński, gm. Korsze.

${ }^{72}$ Równina (niem. Plehnen), pow. kętrzyński, gm. Korsze.

${ }^{73}$ Wypęk, (niem. Weypoth), pow. kętrzyński, gm. Barciany. 
Des kompturs czins

Summarum von dorffern, molen, kretczschmern, huben 200 unde $70 \mathrm{mr}$. guttis geldis.

Summa summarum des pflegers czins zcu Barthen 100 unde $13 \mathrm{mr}$. guttis geldis.

Summa summarum der dinste, der dewtczschen dorffer mit den schultczen 54 dinste.

Summa summarum des kolmischen kornis von den freien 1 last weysen minus $12 \mathrm{sch}$. Item 1 last rocken minus $12 \mathrm{sch}$.

Summa summarum des pluckornis von der heren unde erbarlewthen dorffir 3 leste minus 4 sch. Dy helffte weysen, dy helffte rocken.

Summa summarum der czehenden unde den Prussen.

Item 3 sch. weysen unde 3 leste. Item 3 sch. rocken 3 leste. Item 3 sch. gerste 3 leste. Item 11 leste haber unde 6 sch.

Nadesłany 17 III 2018

Nadesłany po poprawkach 2 XII 2018

Zaakceptowany 6 XII 2018

Dr hab. Rafał Kubicki, prof. UG

Instytut Historii

Uniwersytet Gdański

e-mail:hisrk@univ.gda.pl

ORCID ID: 0000-0002-2171-5713

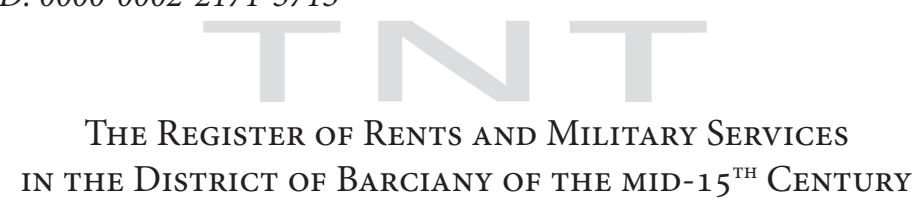

\section{Summary}

Key words: The Teutonic Order in Prussia, economy, settlement, economic sources, registers of rents

The subject of the study is the edition of the list of rents and military services of the Barciany district, including a list of services of the then western part of the Pfleger's territory of Giżycko. This source complements earlier lists of rents from 1422 and 1437, which were issued under the so-called grand book of rent (Das Grosse Zinsbuch des Deutschen Ritterordens). The basis of the edition is the account preserved in Geheimes Staatsarchiv Preußischer Kulturbesitz (Secret Archive of Prussian Cultural Heritage) in Berlin Dahlem (GStA PK), as part of the collection of letters from the former archives of the Teutonic Order (Ordensbriefarchiv No. 28754). The data in- 
cluded in the list may be useful in further work on the reconstruction of the condition of the settlement of Barciany and Kętrzyn in the mid- $15^{\text {th }}$ century. Taking into account previous accounts, thanks to the source presented here, one may trace the changes in the village development and the type, number and distribution of service goods in individual villages.

\section{Ein Verzeichnis der Geld- und Dienstleistungen im Bezirk Barten AUS CA. DER MitTe Des 15. JahrhUNDERTS}

\section{Zusammenfassung}

Schlüsselwörter: Deutscher Orden in Preußen, Wirtschaft, Ansiedlung, Quellen zur Wirtschaft, Zinsverzeichnisse

Gegenstand der Arbeit ist die Edition eines Verzeichnisses von Zinsleistungen und militärischen Diensten aus dem Bezirk Barten (Barciany), das auch eine Zusammenstellung der Dienste im damaligen Westteil der Pflegeamt in Lötzen (Giżycko) umfasst. Diese Quelle bildet eine Ergänzung zu früheren Zinsregistern aus den Jahren 1422 und 1437, die im Rahmen des Großen Zinsbuchs des Deutschen Ritterordens herausgegeben worden sind. Grundlage der Edition ist eine Überlieferung im Geheimen Staatsarchiv Preußischer Kulturbesitz in Berlin-Dahlem (GStA PK), im Bereich der Briefsammlung des früheren Archivs des Deutschen Ordens (Ordensbriefarchiv Nr. 287549. Die in dem Register enthaltenen Daten können bei weiteren zur Rekonstruktion des Siedlungsstands in der Umgebung von Barten und Rastenburg um die Mitte des 15. Jahrhunderts nützlich sein. Wenn man die früheren Register berücksichtigt, kann man auf der Grundlage der hier publizierten Quelle die Veränderungen im Stand der Bewirtschaftung der Dörfer nachverfolgen, und ebenso die Art, Zahl und Verteilung der Dienstgüter auf dem Gebiet einzelner Siedlungen.

\section{BIBLIOGRAFIA}

Beckherrn, Carl. "Beiträge zur Topographie und Statistik des ehemaligen Amtes Rastenburg." Altpreußische Monatsschrift 18 (1881): 398-437, 513-537.

Białuński, Grzegorz. Osadnictwo regionu Wielkich Jezior Mazurskich od XIV do początku XVIII wieku - starostwo leckie (giżyckie) i ryńskie. Olsztyn: Ośrodek Badań Naukowych im. W. Kętrzyńskiego, 1996.

Długokęcki, Wiesław. “Uwagi o genezie i rozwoju wczesnośredniowiecznych Prus do początków XIII w." Pruthenia 2 (2006): 9-54.

Górski, Karol and Marian Arszyński. Barciany. Dzieje zamku i ziemi do połowy XV wieku. Olsztyn: Ośrodek Badań Naukowych im. W. Kętrzyńskiego, 1967.

Joachim, Erich and Walther Hubatsch, eds. Regesta historico-diplomatica Ordinis S. Mariae Theutonicorum 1198-1525, Pars I, vol. 3: (1511-1525). Göttingen: Vandenhoeck \& Ruprecht, 1973. 
Kętrzyński, Wojciech. O ludności polskiej w Prusiech niegdyś krzyżackich. Lwów: Zakład Narodowy im. Ossolińskich, 1882.

Mortensen, Hans and Gertrud Mortensen. Die Besiedlung des nordöstlichen Ostpreußens bis zum Beginn des 17. Jahrhunderts, vol. 1: Die preußisch-deutsche Siedlung am Westrand der Großen Wildnis um 1400. Leipzig: S. Hirzel, 1937.

Rousselle, Martin. "Das Siedlungswerk des Deutschen Ordens im Lande Gerdauen." Altpreußische Forschungen 6 (1929): 220-255.

Swat, Tadeusz. "Dzieje wsi." In Kętrzyn. Z dziejów miasta i okolic, edited by Andrzej Wakar, 161-242. Olsztyn: Pojezierze, 1978.

Tandecki, Janusz and Krzysztof Kopiński. Edytorstwo źródeł historycznych. Warszawa: DiG, 2014.

Thielen, Peter G., ed. Das Grosse Zinsbuch des Deutschen Ritterordens (1414-1438). Marburg: N. G. Elwert, 1958.

Wakuluk, Lidia. "Osadnictwo i sieć parafialna kętrzyńskiego okręgu prokuratorskiego w XIV-XV wieku." Komunikaty Mazursko-Warmińskie (1976) issue 3: 375-395.

Weber, Lothar. Preussen vor 500 Jahren in culturhistorischer, statistischer und militarischer Beziehung nebst Special-Geographie. Danzig: Theodor Bertling, 1878.

Wolff, Adam. "Projekt instrukcji wydawniczej dla pisanych źródeł historycznych do połowy XVI wieku." Studia Źródłoznawcze 1 (1957): 155-184. 